\title{
HEREDITARY POSTERIOR POLAR CATARACT WITH REPORT OF A PEDIGREE*
}

\author{
BY \\ C. G. TULLOH
}

London

A HEREDITARY, developmental, posterior polar cataract has been encountered in a North Country family and its descendants over five generations. It shows two unique features: progression and anticipation.

Although several types of hereditary cataract have already been fully reported in the literature, only one satisfactory pedigree relating to posterior polar cataract has previously been recorded (Ziegler and Griscom, 1915). In the series of cases described in that pedigree, neither of these two features was demonstrated.

\section{Case Reports}

In the family reported here, all except three of those affected in generations II-V were seen personally. The following cases demonstrate the clinical features of the condition:

Case 1 (IV, 11), fcmale, aged 29, was the first patient seen, and the hereditary basis of the cataract was established by tracing her relatives.

She had had good vision as a child, but it had begun to deteriorate noticeably when she was aged about 24. When first seen, at the age of 26 , the visual acuity in the right eye was $6 / 24$ and in the left $6 / 60$ corrected. Posterior polar cataracts were noted, and that in the left eye operated on successfully.

Three years later, she presented asking for the right eye to be dealt with. On examination, the vision had deteriorated to "counting fingers", and there was present a peculiar discoid posterior polar cataract, with fluffy white extensions through the posterior cortex towards the equator. There were also a few anterior cortical punctate opacities (Fig. $1 a, b$, opposite). This eye was successfully operated on also.

There were no other ocular abnormalities.

Her daughter $(V, 15)$, aged $3 \frac{1}{2}$ years, had no cataract.

Case 2 (IV, 7, elder sister of Case 1), female, aged 33, was said to have had cataracts since she was aged about 10 . The vision in both eyes, especially the left, had deteriorated over the past 3 years, and was worse in bright light.

On examination, the visual acuity in the right eye was $6 / 9$ and in the left $6 / 60$ corrected. Both eyes showed a discoid posterior polar opacity, together with fluffy white extensions in the posterior cortex running towards the equator. In addition, there was a small opacity in the anterior cortex of each eye (Fig. $2 a, b$, and $c$, opposite).

*Received for publication March 10, 1955. 


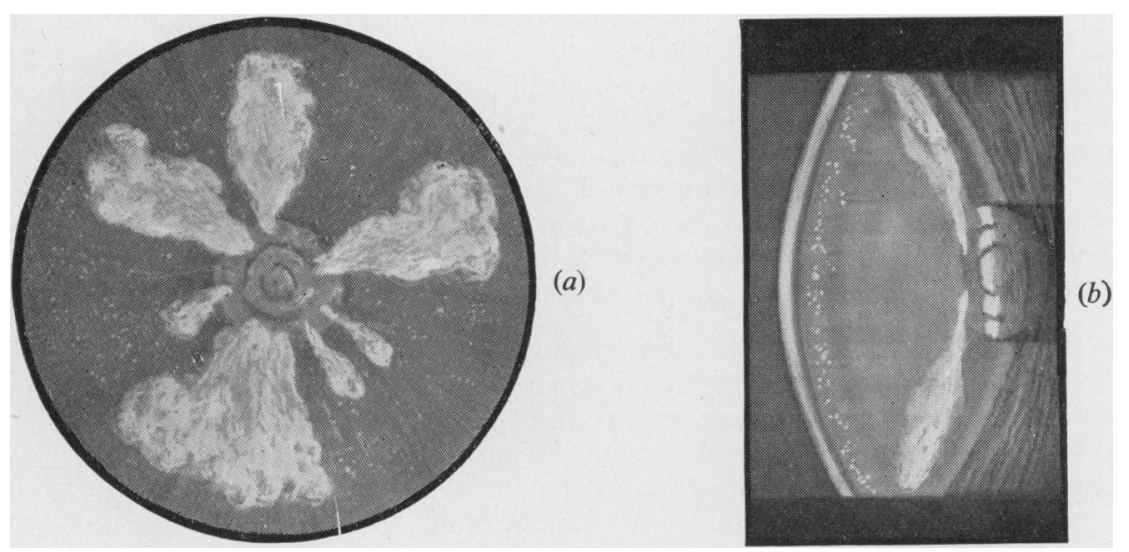

FIG. $1(a)$ and $(b)$.-Case $1(\mathrm{IV}, 11)$

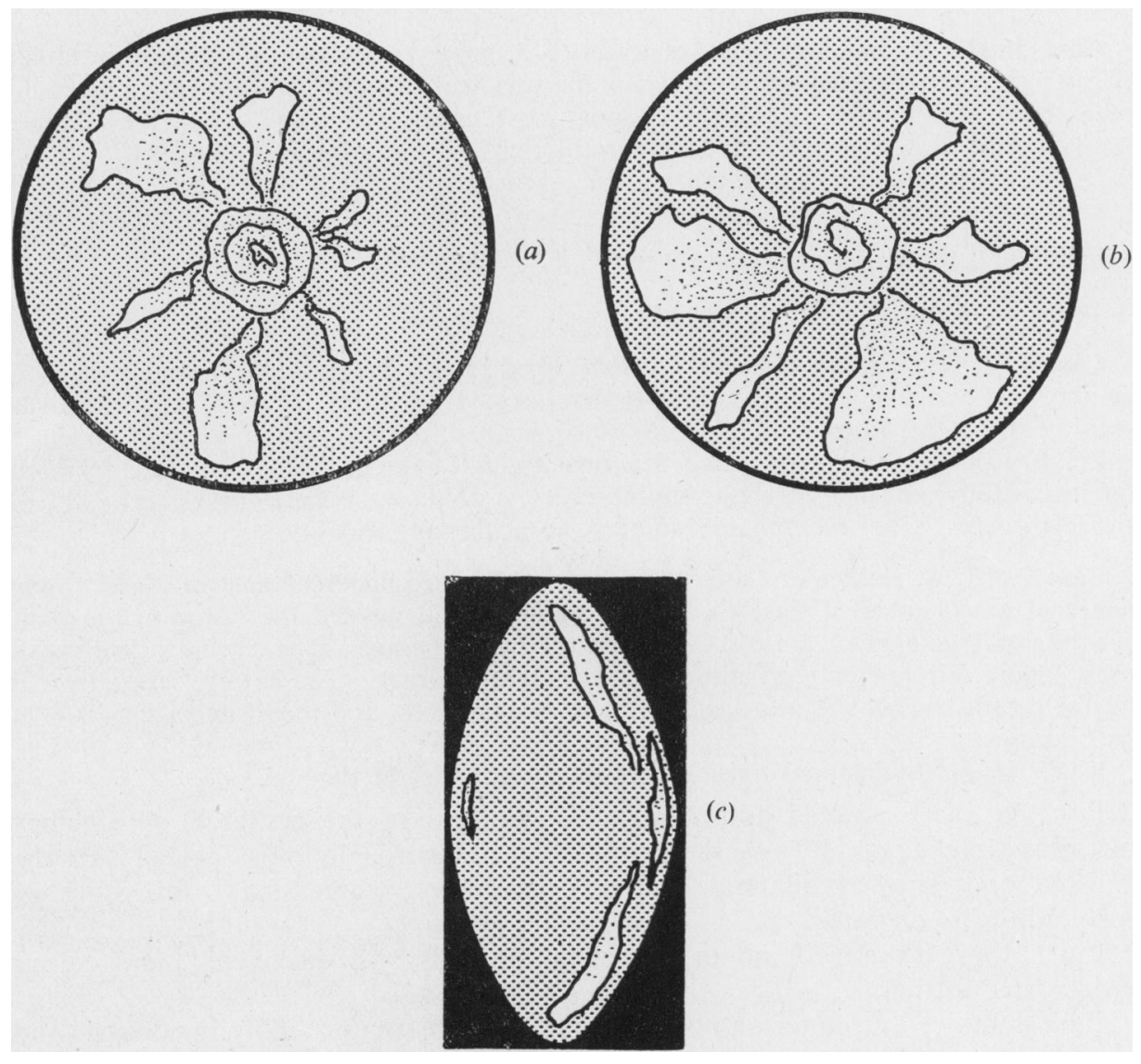

Fig. $2(a, b, c)$.-Case $2(\mathrm{IV}, 7)$. 
Case 3 (IV, 6, elder sister of Cases 1 and 2), female, aged 40, had had good vision when at school, but was found to have posterior polar cataracts when she was 25 years old. At that time, she could not see well in bright sunlight. The cataracts were said to be stationary, but vision deteriorated until operation was called for 5 years ago. The visual acuity in the right eye was $6 / 36$ and in the left $6 / 24$ corrected. The right eye was operated on three times; after the third operation, a severe iritis developed, rendering the eye useless with no perception of light.

The visual acuity in the left eye has remained the same, and this eye shows the typical posterior polar discoid opacity with extensions (Fig. $3 a, b$, opposite).

This patient's daughter $(\mathrm{V}, 11)$ aged 4 years, has no cataract.

Case 4 (IV, 17, first cousin, son of a maternal aunt of Cases 1-3), male, aged 17, was noticed to hold things very close for reading when about 11 years of age. He was seen by an oculist, who said that cataracts were present, but no operation was advised.

On examination, both eyes show the usual discoid posterior polar cataract with extensions, and the visual acuity is $6 / 18$ corrected in each eye. There are also small anterior polar pyramidal cataracts, with reduplication. The opacities are roughly symmetrical in both eyes (Fig. $4 a, b$, and $c$, opposite).

The boy also has some mental aberration, for he was backward at school, and was sent to a special home for 2 years. He is now unemployed. This might be due to his poor visual acuity, but is unlikely, since he can attain N.6 with each eye.

Case 5 (III, 3, maternal aunt of cases 1-3, mother of Case 4), female, aged 44, began to notice visual deterioration when she was aged 20 . Until that time she could read the smallest print. The sight became worse until, at the age of 24 , the left eye was operated on. Since then, the sight in the right eye has continued to fail.

On examination, the right eye shows a mature cataract with visual acuity of " hand movements", accurate projection, and two-point discrimination. The left eye is aphakic, and the visual acuity is $6 / 9$ with $\frac{+10 \cdot 0}{-0.50} 90^{\circ}$.

There is no other ocular abnormality.

Case $6(\mathrm{~V}, 12$, daughter of Case 2), female, aged 11, was first noted to have poor vision at about $2 \frac{1}{2}$ years of age. It gradually deteriorated until the age of 4 , when she was seen by an oculist, who noted bilateral, saucer-shaped, posterior polar cataract, together with a few anterior and posterior cortical opacities in each eye. Both eyes were operated on successfully, and her corrected visual acuity is now 6/9 in the right eye and 6/12 in the left. Apart from the aphakia, there is no ocular abnormality.

Case 7 (III, 1, mother of Cases 1-3, sister of Case 5, maternal aunt of Case 4, and maternal grandmother of Case 6), female, aged 64, had noticed the vision in the right eye gradually deteriorate since she was about 20 years of age. She can now distinguish only " hand movements", on account of a mature cataract. The left eye was operated on for cataract when she was aged 46; it is now aphakic and the visual acuity is $6 / 24$ with glasses.

When young, this patient's vision was always poor in the daytime.

For the other affected and unaffected members of the pedigree, satisfactory histories were available, together with clinical records in most cases. All the affected ones showed bilateral cataracts, gradually progressing from youth or early adult life onwards.

In all those examined, no other ocular anomaly was discovered, and colour vision was normal.

Case 4 showed an anterior polar cataract, which was probably incidental, and also some mental aberration. 

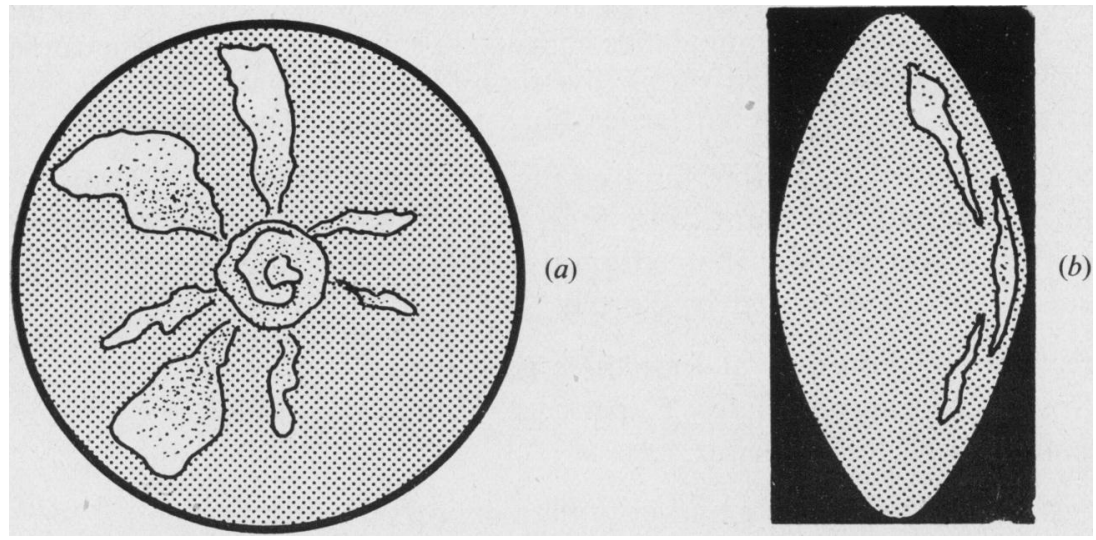

FIG. $3(a, b)$.-Case $3(\mathrm{IV}, 6)$.
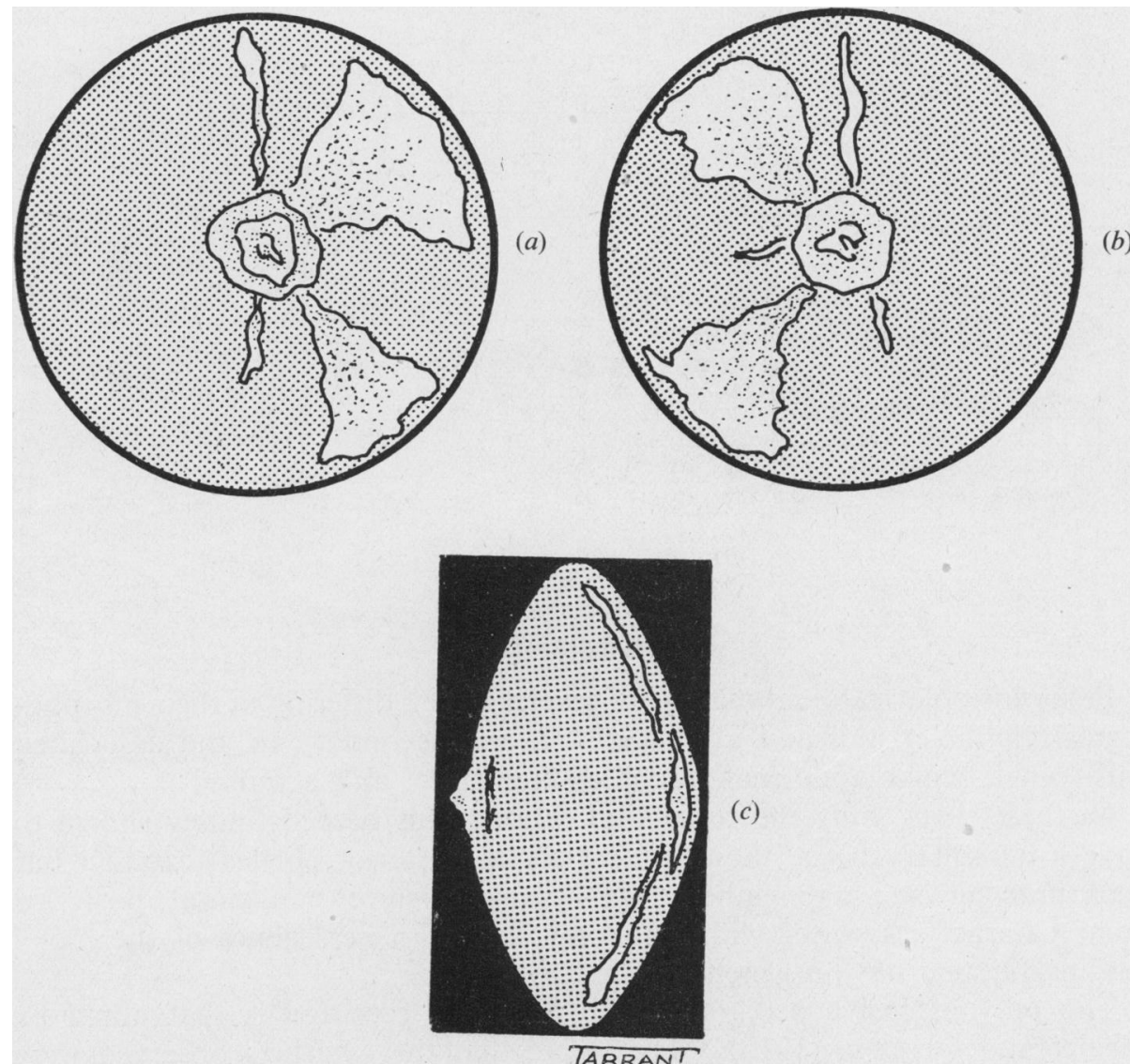

(c)

FIG. $4(a, b, c)$.-Case 4 (IV, 17). 
One unaffected case showed high myopia in one eye, with $a$ divergent squint, whilst another showed an accommodative convergent squint due to hypermetropia.

There was no evidence of a myotonic dystrophy or its associated features, and the social position of the diffèrent generations has remained unchanged.

The genealogical tree of this family (Fig. 5) reveals that the pathogenic gene is an autosomal dominant, and that the original individual known to manifest the condition was heterozygous. It satisfies the criteria of an autosomal dominant as noted by Sorsby (1951):

(a) direct transmission over at least three generations;

(b) a frequency of approximately 50 per cent. of individuals affected;

(c) no predilection for either sex.

There is normal penetrance and complete expression of the gene.

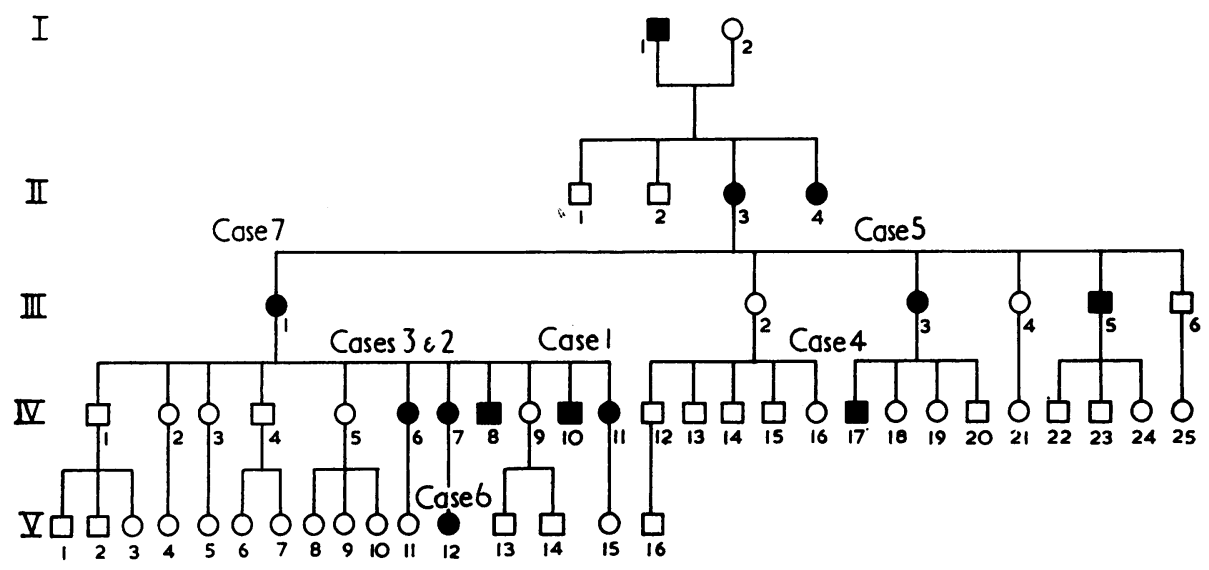

FIG. 5.-Pedigree chart.

\section{Discussion}

Posterior polar cataract may be of several types, differing in their morphological characteristics and age incidence, and secondary to and associated with other ocular abnormalities, both congenital and acquired.

Of these types, only the congenital cataract has been definitely shown to have a hereditary basis, though a pre-senile posterior cortical cataract has been noted in several members of the same sibship; congenital posterior polar cataract associated with lenticonus and. with persistence of the foetal vascular system has no genetic origin.

The only extensive pedigree which has been reported is that noted by Ziegler and Griscom (1915). They state that the cataracts observed were congenital, but not whether the patients were seen in infancy; it is not 
possible to say that the opacities were present at birth in such cases merely from their morphological characteristics. In addition, it is not stated whether the cataracts were stationary or progressive.

In the present series, the morphology of the first formed posterior polar opacities is similar to that described by Ziegler and Griscom, namely, a roughly circular disk covering the central quarter of the posterior cortex and consisting of two or three concentric rings of opacity. The extensions arise from the anterior surface of this disc, and radiate forwards and outwards towards the equator, parallel with the posterior lens capsule. They consist of fluffy white masses developing in the lens fibres. In one case, a bilateral anterior polar pyramidal cataract with reduplication was also noted, and some cases had a few punctate opacities scattered throughout the cortex.

The age at which progression began in the present series of cases is a most interesting feature, and might be said to demonstrate the phenomenon of anticipation. In the third generation, appreciable visual loss did not begin until adult life, but in the fourth generation it began between puberty and adolescence, and in the fifth generation in childhood.

It is suggested, therefore, that the original posterior polar opacities were congenital, but progressed by means of the " extensions " already described, the progression beginning at an earlier age in each succeeding generation.

\section{Summary}

A unique type of hereditary, developmental, posterior polar cataract of a progressive nature is described, with the report of a pedigree.

\section{REFERENCES}

SorSBY, A. (1951). “Genetics in Ophthalmology”, p. 7. Butterworth, London. ZIEGLER, S. L., and Griscom, J. M. (1915). Trans. Amer. ophthal. Soc., 14, 356. 